\title{
TRANSTORNOS DE APRENDIZAGEM: em foco a Dislexia
}

Ana Claudia Gonçalves Araújo ${ }^{1}$

\section{RESUMO}

O presente estudo trata-se de uma revisão bibliográfica sobre os transtornos de aprendizagem, tendo como foco a Dislexia. Para a sua realização tomamos como base livros e artigos que abarcam a temática. Nosso objetivo foi conhecer melhor sobre os transtornos de aprendizagem, como identifica-los dentro das escolas e de que maneira realizar intervenções. Em nossa investigação localizamos distinções nas terminologias e na origem da Dislexia. Percebeu-se que o diagnóstico precoce é a melhor forma para evitar problemas futuros. Entretanto, para isso, vê-se como necessárias ações da escola para a formação dos professores; a melhoria na formação inicial e a autoformação docente; e a participação efetiva da família no processo de ensino e aprendizagem.

Palavras-chave: Transtornos de Aprendizagem. Dislexia. Formação Inicial e Continuada.

\section{ABSTRACT}

The present study is a bibliographic review on learning disorders, focusing on Dyslexia. For its realization we take as a base books and articles that cover the theme. Our goal was to learn more about learning disorders, how to identify them within schools and how to carry out interventions. In our investigation, we found distinctions in the terminology and origin of Dyslexia. It was realized that early diagnosis is the best way to avoid future problems. However, for this, it is seen as necessary school actions for the training of teachers; improvement in initial training and teacher self-training; and the effective participation of the family in the teaching and learning process.

Keywords: Learning Disorders. Dyslexia. Initial and Continuing Formation.

\section{INTRODUÇÃO}

Os transtornos de aprendizagem sempre fizeram parte do processo de ensino e aprendizagem de pessoas do mundo todo. O problema é que durante centenas de anos os educadores não tinham o conhecimento dessas distinções neurobiológicas que interferiam na capacidade de leitura, escrita, na linguagem e em raciocínio matemático dos estudantes.

\footnotetext{
${ }^{1}$ Graduação em Pedagogia (Faculdade de Caldas Novas, 2011); Pós-graduação em Psicopedagogia Institucional e Clínica (FABEC, 2012) e Gestão Educacional (APOGEU, 2013); Mestranda em Ciências da Educação (UNADES). E-mail: anaclaudiapsci@gmail.com
} 
$\mathrm{Na}$ atualidade, a temática dos transtornos de aprendizagem, em especial da Dislexia, deveria ser corriqueira no ambiente familiar e escolar. O que se percebe é a ausência de conhecimento por parte de escola, professores e responsáveis dos alunos, ou ainda, uma noção básica desses distúrbios, sem saber como identificá-los ou intervir sobre eles.

As crianças em idade escolar são as mais afetadas pelos problemas que um transtorno de aprendizagem pode acarretar. Além de dificultar o processo de aprendizagem das disciplinas curriculares, esses distúrbios podem ser associados à hiperatividade, falta de atenção, sentimentos de inferioridade, irritabilidade, dificuldades de socialização, e ainda, podem sofrer bullying por não se enquadrarem nos padrões de normalidade da sociedade.

Diante da importância que a temática assume no âmbito acadêmico, científico e social, nos dispusemos a aprofundar sobre ela. Nosso objetivo foi conhecer melhor sobre os transtornos de aprendizagem, como identifica-los dentro das escolas e de que maneira realizar intervenções. Trata-se, portanto, de uma revisão bibliográfica sobre os transtornos de aprendizagem, tendo como foco a Dislexia. Para a sua realização tomamos como base livros e artigos que abarcam a temática.

\section{TRANSTORNOS DE APRENDIZAGEM E A DISLEXIA}

Entende-se como Transtornos de Aprendizagem, Problemas de Aprendizagem, Distúrbios de Aprendizagem ou Dificuldades de Aprendizagens (terminologias também encontradas na literatura) os acometimentos das habilidades específicas de um indivíduo durante o seu processo de aprendizagem. Eles podem afetar e dificultar $o$ desenvolvimento intelectual, escolar ou acadêmico, profissional e até mesmo social das pessoas que o apresentam (FLETCHER, 2009).

O Manual Diagnóstico e Estatístico de Transtornos Mentais (DSM-V), sigla em inglês para Diagnostic and Statistical Manual of Mental Disorders, considera esses distúrbios como transtornos do neurodesenvolvimento. Geralmente eles aparecem bem cedo, ainda na infância, antes dela ingressar na escola, entretanto, dificilmente os familiares notam seus sintomas (LEAL; NOGUEIRA, 2012). 
Transtornos de aprendizagem afetam a capacidade de o indivíduo aprender em várias áreas, não apenas conteúdos escolares. Isso porque eles consistem tanto em dificuldades no processo de aprendizagem quanto em alterações comportamentais. Rotta, Ohlweiler e Riesco (2016) exemplificam que uma pessoa com dificuldade para ler e/ou escrever pode ter problemas relacionados à: socialização, memória, coordenação motora e atividades mentais alteradas.

Os autores supracitados alertam para um mito nessa área, o de que os transtornos de aprendizagem afetam apenas crianças. Adultos também podem apresentar essas condições em grau menor ou semelhante ao das crianças (FLETCHER, 2009). Essa impressão de que apenas crianças apresentam esses problemas é devido ao fato de que os adultos, na atualidade, não puderam ser diagnosticados ainda na infância.

Não há consenso sobre as causas dos transtornos de aprendizagem. O pressuposto mais aceito é o de que tenham origem biológica. Entretanto, Teixeira e Alliprandini (2013) acrescentam que os fatores sociais, ambientais, circunstanciais e pedagógicos também podem influenciar no seu surgimento ou desenvolvimento dos problemas de aprendizagem.

Os manuais de diagnóstico trazem alguns pontos que os especialistas não devem levar em consideração como consequência dos transtornos de aprendizagem: a) doença cerebral ou traumatismo; b) algum comprometimento visual ou auditivo que não foi corrigido; c) comprometimento na inteligência global; d) falta de oportunidade em aprender; e) mudança de escola ou de professor (NEURO SABER, 2018). Nesse caso, deve-se avaliar a criança como um todo antes de emitir qualquer tipo de posição diagnóstica.

Há indícios de que os transtornos de aprendizagem tenham uma relação com a gravidez. Isso porque, durante uma gestação, são construídas interligações de informações em determinadas regiões cerebrais, causando os distúrbios, ou seja, interferem no desenvolvimento cerebral do feto. Seja por problemas durante a gestação ou o parto, ou por limitações de oportunidade na escola, ou sofrimento com o bullying, viver em um ambiente familiar desfavorável e, até mesmo a metodologia de ensino das escolas, todos são elementos que contribuem com o aparecimento ou evolução dos transtornos (TEIXEIRA; ALLIPRANDINI, 2013). 
Lehmkuhl (2015) alerta para o papel do professor e da família ao identificar os primeiros sintomas dos transtornos de aprendizagem. Muitas vezes, os responsáveis acreditam que a criança tem distúrbio de aprendizagem, quando na verdade simplesmente não está confortável com o ambiente escolar ou com a metodologia do professor. É preciso, portanto, que os familiares mantenham um contato direto com a escola e, da mesma forma, a escola com a família.

O Manual Diagnóstico e Estatístico de Transtornos Mentais (DSM-V) classifica os distúrbios de aprendizagem em três categorias: a) transtorno com prejuízo na leitura; b) transtorno com prejuízo na matemática b) transtorno com prejuízo na escrita. Para além disso, deve-se compreender que há mais distúrbios que podem afetar gravemente a capacidade de aprender das crianças

A exemplo disso, o Manual Diagnóstico e Estatístico de Transtornos Mentais (DSM-V) abrange algumas especificidades dos distúrbios de aprendizagem, e por isso, os especialistas utilizam o CID 10 (fornece códigos relativos à classificação de doenças e de uma grande variedade de sinais, sintomas, aspectos anormais, queixas, circunstâncias sociais e causas externas para ferimentos ou doenças). Exemplos: CID10R90 Transtornos relacionados à atenção, CID10-R48 transtornos da Dislexia, CID10-F80 transtornos da linguagem, CID10-F81 transtornos de habilidades escolares.

O Transtorno de Déficit de Atenção e Hiperatividade (TDAH) é um dos transtornos de aprendizagem mais conhecidos entre pais e educadores. DuPaul e Stoner (2007) classificam esse distúrbio como dificuldade de concentração e, por isso, não consegue fazer tarefas e trabalhos escolares; exibem mudanças comportamentais; apresentam-se ansiosos, inquietos e podem até mesmo serem agressivos.

Ainda segundo os autores, devido à falta de concentração, as crianças com TDAH parecem ser teimosas ou irredutíveis. Têm dificuldades para seguirem instruções, muitas vezes simples; não sabem esperar sua vez nas brincadeiras e atividades escolares; fazem as avaliações muito rápido, e por isso, erram muitas coisas; apresentam notas baixas apesar de, muitas vezes, saberem o conteúdo; estão sempre em conflito com colegas, professores ou pais. A Associação Brasileira de Déficit de Atenção (Abda) explica que esses sujeitos apresentam sintomas de desatenção, inquietude e impulsividade. 
O TDAH pode acompanhar as crianças pela vida toda, atrapalhando o rendimento tanto nos estudos quanto no trabalho. Portanto, em nossa sociedade atual há vários adultos com esse transtorno de aprendizagem que nem sabem que o tem. É preciso que se fique atento aos sinais, quanto mais cedo for identificado e tratado, menos consequências ele acarretará para a vida da pessoa.

A Discalculia, como o próprio nome indica, dificulta a aprendizagem da matemática ou cálculos. Os sujeitos que apresentam esse distúrbio não conseguem compreender o raciocínio por trás das operações matemáticas, têm um senso numérico fraco e ficam ansiosos quando precisam fazer exercícios dessa disciplina na escola, o que acaba acarretando em mais dificuldades (FLETCHER, 2009).

Nas crianças muito pequenas percebe-se a dificuldade de lembrar dos números, não conseguir ler as horas, ficam perdidos nos livros (capítulos, páginas e número de questões). Nos adolescentes, não conseguem medir ingredientes em uma receita, interpretar informações de gráficos e/ou tabelas, ou ainda, calcular um troco. Geralmente a Discalculia vem associada à Dislexia que trataremos mais à frente (LEAL; NOGUEIRA, 2012).

A disgrafia afeta a habilidade de escrita do sujeito. No caso das crianças, elas apresentam problemas quando começam a escrever as primeiras letras e/ou números. Pode se apresentar tanto de uma caligrafia ilegível (diferente de feia) e da escrita errada de palavras. Devido às suas dificuldades os alunos que têm esse distúrbio são lentos para finalizar atividades escolares (ROTTA; OHLWEILER; RIESCO, 2016).

Os mesmos autores citados anteriormente explicam que há dois tipos de Disgrafia. A primeira, a motora, se caracteriza pela dificuldade motora no momento de escrever. Já a segunda, a perceptiva, a pessoa pode dominar a leitura e a oralidade, mas não consegue escrever as palavras corretamente. Teixeira e Alliprandini (2013) alertam que um dos primeiros sinais desse transtorno de aprendizagem na escola é a inabilidade da criança em participar de ditados de palavras, ainda nas séries iniciais.

Outro distúrbio de aprendizagem bastante conhecido, na atualidade, é a Dislexia. A Dislexia atinge cerca de 15 milhões de brasileiros, e a Associação Brasileira de Dislexia $(A B D)$ estima que esse distúrbio seja o de maior incidência nas salas de aula, e hoje, abrange $5 \%$ e $17 \%$ da população mundial. Entretanto, a $A B D$, em pesquisa, alerta que 
60\% dos casos avaliados (2013 a 2018) não eram disléxicos, $67 \%$ são do sexo masculino, $81 \%$ têm antecedentes familiares, e $69 \%$ apresentou alterações nos exames de processamento auditivo.

A Dislexia é caracterizada, sobretudo, pela dificuldade em ler e em escrever. Pessoas disléxicas demoram ou possuem problemas para reter informações e compreender conceitos linguísticos simples. A criança, troca letras de lugar, enxerga as letras espelhadas, se confunde com os sons e não consegue reproduzir o que escuta na escrita. Esse transtorno de aprendizagem tem uma origem neurológica e, na maioria das vezes, em meninos por hereditariedade (DAVIS, 2004).

Nas pessoas com Dislexia a capacidade de decodificação de signos linguísticos e de soletração é bem reduzida e/ou lenta. Geralmente, as crianças apresentam também a falta de atenção, sendo esta, bastante comum nesse tipo de transtorno de aprendizagem. Embora o seu processo de aprendizagem seja diferente, o aluno com esse distúrbio não é menos inteligente que os demais, apenas lhe exigirá um esforço maior para aprender. Há registros de disléxicos que se destacam muito nas exatas e na arte (FLETCHER, 2009).

A criança ou o adulto com Dislexia sofre muito preconceito, as pessoas à sua volta não conseguem entender suas limitações e necessidades educacionais. Como para aprender exige dela muito esforço isso pode ocasionar em muitas situações constrangedoras no ambiente escolar. Devido a isso, muitas crianças disléxicas acabam perdendo o interesse pela escola e abandonando-a precocemente. Por isso, Costa e Crenitte (2017) sugerem que os professores e a família incentivem as crianças a estudarem com muita paciência, com respeito às suas próprias limitações, e com a ajuda de um profissional, como um psicopedagogo.

As autoras supracitadas acreditam ainda que quando qualquer problema acontece no processo de aprendizagem, os pais, geralmente, tendem a se preocupar em excesso ou não compreender o motivo de tanta dificuldade nas atividades escolares. Em ambos os casos, a criança sempre sair perdendo. As pesquisadoras sugerem que para ajudar os alunos a superarem os obstáculos que dificultam a leitura e a escrita, os pais e os professores precisam estar cientes dos sinais da Dislexia, bem como, das possibilidades de intervenção. 
Há critérios que devem ser levados em consideração para se fazer um diagnóstico da Dislexia. Primeiramente se encaminha para um médico (clínico geral) que diante da anamnese e exames a encaminhará para um neurologista. Diante da negativa de problemas neurológicos físicos, este será encaminhado para um fonoaudiólogo, neuropsicólogo e/ou psicopedagogo. Esse encaminhamento sempre dependerá da queixa da família, de relatórios emitidos pela escola, e dos resultados dos exames realizados (LEAL; NOGUEIRA, 2012).

O ideal é que esse diagnóstico nunca seja "fechado" por um único profissional, o trabalho em equipe multidisciplinar é de suma importância. Quatro macro-critérios devem ser avaliados minuciosamente: fala, linguagem, estado psicológico e funções cognitivas. Posteriormente averiguar se as dificuldades excedem 6 meses, depois constatar se a criança apresenta dificuldades relacionadas a: a) grafia; b) compreender o significado do conteúdo; c) escrita; d) leitura (lenta ou imprecisa); e) dominar o sentido dos números; f) pensar matematicamente (ROTTA; OHLWEILER; RIESCO, 2016).

A idade da criança, o histórico familiar e escolar, devem ser considerados oportunos para um diagnóstico. Os transtornos que afetam a aprendizagem danificam a sua autoestima, vida social, relacionamento familiar, entre outros. Nesse caso, é preciso leva-los em consideração, também, pois crianças excessivamente tímidas ou irrequietas podem não conseguir se socializar adequadamente (TEIXEIRA; ALLIPRANDINI, 2013).

Muitas crianças sofrem por não conseguir socializar com os colegas de turma, têm medo de ficar longe dos pais, sentem-se irritadas por levar muito tempo para terminar tarefas domésticas e vergonhadas por demorar a copiar a tarefa na escola. Por isso, é preciso que o "tratamento" envolva quadro medidas: a) intervenção psicopedagógica; b) intervenção fonoaudiológica; c) programas educativos especiais (aulas de reforço e Atendimento Educacional Especializado (AEE); d) uso de medicamentos para os casos mais sérios (COSTA; CRENITTE, 2017).

Vale ressaltar que a psicopedagogia atua no estudo e intervenção da aprendizagem humana. É um campo do conhecimento que mescla as ciências: psicologia e pedagogia, mas para identificar falhas no processo de ensino ela utiliza dos conhecimentos: da antropologia, da psicanálise, da neurologia, da fonoaudiologia e da psicolinguística. O profissional dessa área "psicopedagogo" é qualificado para organizar: 
planos de estudo; aliviar sintomas desagradáveis de condições que não têm cura; corrigir comportamentos em relação ao período reservado para estudar; ou seja, diagnosticar e intervir (WEISS, 2002).

O primeiro passo para a identificação de um dos transtornos de aprendizagem é conhecê-los. É preciso, portanto, que os professores sejam capazes de notar em seus alunos a presença dos primeiros sintomas. Bispo (2016), em sua pesquisa com docentes de séries iniciais, notou que nos excertos dos envolvidos enfatizavam uma formação incompleta, marcada pela ausência da relação entre teoria e prática, não possuindo qualquer conhecimento da extensão do problema envolvendo as dificuldades de aprendizagem.

Outro item bastante discutido na pesquisa da autora supracitada foi a ausência da família no processo de ensino e aprendizagem de seus filhos. Somado a isso, a escassez de ações das escolas em promover uma formação continuada aos docentes, nessa área, a fim de atender a demanda dessas crianças. Podemos notar, portanto, que é preciso que haja um esforço conjunto entre família, escola e professores para mudar a realidade desses estudantes.

\section{CONSIDERAÇÕES FINAIS}

O processo de ensino não pode ser visto como um momento de transmissão de conteúdo, ao contrário, é a chance de estimular, dirigir, incentivar, impulsionar o processo de aprendizagem de qualquer sujeito. Portanto, enxergando-o como uma relação recíproca entre docente e discente, passamos a entrever de forma mais clara a responsabilidade dos professores no aprender dos alunos.

No caso dos transtornos de aprendizagem, em especial a Dislexia, não é diferente. O professor tem um papel primordial, já que é na escola que aparecem os primeiros sintomas. Além disso, espera-se que esses profissionais conheçam esses distúrbios e sejam capazes de identificar seus primeiros sintomas, visto que o diagnóstico precoce, é importante.

Como a quantidade de pessoas com Dislexia tem aumentado significativamente a cada ano, é preciso que as escolas tomem medidas para lidar com esses distúrbios. 
Oferecer cursos de formação continuada (teoria e prática) para os profissionais da educação, e também, conduzir as famílias ao conhecimento. Isso pode ser feito realizando reuniões, ofertando palestras e/ou encaminhando a profissionais especializados.

\section{REFERÊNCIAS}

ABD. Associação Brasileira de Dislexia. Estatísticas 2013-2018. Disponível em: $<$ http://www.dislexia.org.br/wp-content/uploads/2017/10/Estat\%C3\%ADsticas-

Disl\%C3\%A9xicos-avaliados-entre-2013-e-2018-ABD.pdf>. Acesso em: 14 jan. 2018.

BISPO, Silvana Alves da. VASCONCELOS, Maria Lucia Marcondes Carvalho. Educação Humanizadora e Dificuldades de Aprendizagem: o que nos revelam os discursos de professores dos anos iniciais do Ensino Fundamental? Universidade Presbiteriana Mackenzie, São Paulo, 2016.

COSTA, A. L. A.; CRENITTE, P. A. P. Formação Docente: programa de atualização voltado para ações com alunos com distúrbios específicos de aprendizagem. Bauru - SP, 2017.

DAVIS, Ronald D. O Dom da Dislexia. 1. ed. Rio de Janeiro/RJ: Editora Rocco, 2004.

DUPAUL, George J.; STONER, Gary. TDAH nas Escolas: estratégias de avaliação e intervenção. 1. ed. São Paulo/SP: M. Books, 2007.

FLETCHER, Jack M. Transtornos de Aprendizagem: da identificação à intervenção. Porto Alegre: Penso, 2009.

GIL, A. C. Métodos e Técnicas de Pesquisa Social. São Paulo: Atlas, 1994.

LEAL, D.; NOGUEIRA, M, O, G. Dificuldades de aprendizagem um olhar psicopedagógico. Curitiba: Intersaberes, 2012.

LEHMKUHL, Márcia de Souza. Formação continuada de professores na área de educação especial. $37^{\circ}$ Reunião Nacional da ANPED, UFSC - Florianópolis, out/2015.

NEURO SABER. O que são Transtornos de Aprendizagem? 2018. Disponível em: https://institutoneurosaber.com.br/o-que-sao-transtornos-de-aprendizagem/. Acesso em: 18 jan. 2018

ROTTA, N. T.; OHLWEILER, L.; RIESCO, R. dos S. Transtornos da Aprendizagem: abordagem neurobiológica e multidisciplinar. Porto Alegre: Artmed, 2016. 
TEIXEIRA, Andrea Regina; ALLIPRANDINI, Paula Mariza Zedu. Intervenção no uso de estratégias de aprendizagem diante de dificuldades de aprendizagem. Revista Semestral da Associação Brasileira de Psicologia Escolar e Educacional, São Paulo - SP, Vol 17, no 2, Jul/Dez 2013: p. 279-288.

WEISS, Maria Lúcia. Psicopedagogia Clínica: uma visão diagnóstica dos problemas de aprendizagem escolar. 9. ed. Rio de Janeiro: DP\&A editora, 2002. 